\title{
ON $J$-SELFADJOINT EXTENSIONS OF $J$-SYMMETRIC OPERATORS
}

\author{
IAN KNOWLES
}

\begin{abstract}
A short proof is given (via the theory of conjugate-linear operators) of the fact that every $J$-symmetric operator in a Hilbert space $\mathcal{X}$ has a $J$-selfadjoint extension in $\mathcal{H}$.
\end{abstract}

An operator $J$ on a complex Hilbert space $\mathcal{H}$ is called a conjugation if it is an involution and

$$
(J x, J y)=(y, x)
$$

for all $x$ and $y$ in $\mathcal{H}$. Following I. M. Glazman [3], a linear operator $A$ in $\mathcal{H}$ with domain $\mathscr{D}(A)$ dense in $\mathcal{H}$ is called $J$-symmetric if

$$
(A x, J y)=(x, J A y)
$$

for all $x$ and $y$ in $\mathscr{D}(A)$. Clearly, $A$ is $J$-symmetric if and only if $J A J \subset A^{*}$. If $J A J=A^{*}$, then $A$ is said to be $J$-selfadjoint.

Now, it is well known that symmetric linear operators in $\mathcal{H}$ need not possess selfadjoint extensions; indeed, it is a basic fact in the theory that these operators are precisely those with unequal deficiency indices (see [1, Corollary 13, p. 1230]). It is thus rather surprising to learn that the following result is known for $J$-symmetric operators. $\mathcal{H}$.

THEOREM A [2]. Every J-symmetric operator in $\mathcal{H}$ has a J-selfadjoint extension in

The method of proof used in [2] makes use of certain properties of the graphs of the operators concerned. We return to this method later. Our main objective here is to give a simplified proof of this result.

The method that we use is based upon a consideration of the analogous theory for conjugate-linear operators in $\mathcal{H}$. Here, an operator $T$, with domain $\mathscr{D}(T)$ dense in $\mathcal{K}$, is said to be conjugate-linear if

$$
T(\alpha x+\beta y)=\bar{\alpha} T x+\bar{\beta} T y
$$

for all scalars $\alpha$ and $\beta$, and all $x$ and $y$ in $\mathscr{D}(T)$. By analogy with the corresponding theory for linear operators, we define $\mathscr{D}\left(T^{*}\right)$ to be the set of elements $y$ in $\mathcal{H}$ for which there corresponds an element $z$ in $\mathcal{H}$ (necessarily unique) such that $(T x, y)$ $=(z, x)$ holds for all $x$ in $\mathscr{D}(T)$. The adjoint, $T^{*}$, of $T$ is defined on $\mathscr{D}\left(T^{*}\right)$ by the equation $T^{*} y=z$. Thus

Received by the editors April 18, 1979.

AMS (MOS) subject classifications (1970). Primary 47B25.

$K e y$ words and phrases. $J$-symmetric operators, $J$-selfadjoint extension, conjugate-linear operator. 


$$
(T x, y)=\left(T^{*} y, x\right)
$$

for all $x \in \mathscr{D}(T)$ and $y \in \mathscr{D}\left(T^{*}\right)$. The conjugate-linear operator $T$ is said to be symmetric if

$$
(T x, y)=(T y, x)
$$

for all $x$ and $y$ in $\mathscr{Q}(T)$; thus $T$ is symmetric if and only if $T \subset T^{*}$. If $T=T^{*}$, then $T$ is called selfadjoint. Finally, we say that $T$ is maximal symmetric if $T$ is symmetric and possesses no proper symmetric extensions. The usual maximality argument, using Zorn's lemma, shows that every symmetric conjugate-linear operator has a maximal symmetric extension. Thus, if we observe that the linear operator $A$ is $J$-symmetric ( $J$-selfadjoint) if and only if the conjugate-linear operator $J A$ is symmetric (selfadjoint), then Theorem A may be equivalently formulated as,

THEOREM B. Every maximal symmetric conjugate-linear operator is selfadjoint.

Proof. If we assume to the contrary, that the theorem is not true, then there is a conjugate-linear operator $T$ that is maximal symmetric, but not selfadjoint.

Let $y \in \mathscr{D}\left(T^{*}\right) \backslash \mathscr{D}(T)$, and define an extenson $T_{1}$ of $T$ by

$$
\mathscr{D}\left(T_{1}\right)=\mathscr{D}(T)+\{\alpha y\}, \quad \alpha \in \mathbf{C},
$$

and, for $x=p+q \in \mathscr{D}\left(T_{1}\right)$ where $p \in \mathscr{D}(T)$ and $q \in\{\alpha y\}$ set

$$
T_{1} x=T p+T^{*} q \text {. }
$$

Then, for $x=p_{1}+q_{1}$ and $y=p_{2}+q_{2}$ in $\mathscr{D}\left(T_{1}\right)$, where $q_{1}=\alpha_{1} y$ and $q_{2}=\alpha_{2} y$ for some complex scalars $\alpha_{1}$ and $\alpha_{2}$, we have

$$
\begin{aligned}
\left(T_{1} x, y\right) & =\left(T p_{1}, p_{2}\right)+\left(T p_{1}, q_{2}\right)+\left(T^{*} q_{1}, p_{2}\right)+\left(T^{*} q_{1}, q_{2}\right) \\
& =\left(T p_{2}, p_{1}\right)+\left(T^{*} q_{2}, p_{1}\right)+\left(T p_{2}, q_{1}\right)+\bar{\alpha}_{1} \bar{\alpha}_{2}\left(T^{*} y, y\right) \\
& =\left(T_{1} y, x\right) .
\end{aligned}
$$

Thus $T_{1}$ is a proper symmetric extension of $T$, in contradiction to the maximality of $T$. The proof is complete.

As a final result we have:

Theorem C. Let $\mathcal{H}_{2}=\mathcal{H} \oplus \mathcal{H}$, and let $G_{A}=\left\{[x, y] \in \mathcal{H}_{2}: y=A x\right\}$ denote the graph of the closed J-symmetric operator A. Let $T: \mathcal{H}_{2} \rightarrow \mathcal{H}_{2}$ be defined by $T[x, y]$ $=[J y,-J x]$, and set $D=\mathscr{H}_{2} \ominus\left[G_{A} \oplus T G_{A}\right]$. Then

(i) there exists a subspace $X$ in $\mathscr{K}_{2}$ such that $X \oplus T X=D$, and the set $G_{A} \oplus X$ is the graph of a $J$-selfadjoint extension of $A$,

(ii) every $J$-selfadjoint extension of $A$ has the form given in (i).

Proof. Part (i) is precisely the result proved in [2]. Alternatively, part (i) may be derived from Theorem $A$, and part (ii), which we now prove. Let $B$ be an arbitrary $J$-selfadjoint extension of $A$, with graph $G_{B}$, and set $X=G_{B} \ominus G_{A}$. Since $G_{J B^{*} J}=$ $T G_{B}^{\perp}$, and $B$ is $J$-selfadjoint, it is clear that $G_{B} \oplus T G_{B}=\mathcal{H}_{2}$, and thus that

$$
X \oplus G_{A} \oplus T\left(X \oplus G_{A}\right)=\mathcal{K}_{2} \text {. }
$$


Finally, as $X \perp G_{A}$, it is not hard to see that $T X \perp T G_{A}$. The result now follows from (5).

\section{REFERENCES}

1. N. Dunford and J. T. Schwartz, Linear operators. Part II. Spectral theory, Interscience, New York, 1963.

2. A. Galindo, On the existence of $J$-selfadjoint extensions of J-symmetric operators with adjoint, Comm. Pure Appl. Math. 15 (1963), 423-425.

3. I. M. Glazman, An analogue of the extension theory of hermitian operators and of the non-symmetric one-dimensional boundary-value problem on a semi-axis, Dokl. Akad. Nauk SSSR 115 (1957), 214-216.

Department of Mathematics, University of California at Berkeley, Berkeley, California 94720

Department of Mathematics, Universtty of the WtTwatersrand, Johannesburg, SOUth Africa 